Andrea D'Urso

\title{
Bounoure, Effenberger et les « réflexions parallèles ॥ de La civilisation surréaliste ou la sémiotique du surréalisme après Breton et Teige
}

Résumé: Notre contribution montrera, sans doute pour la toute première fois, l'intérêt des réflexions sémiotiques des poètes surréalistes tchèques et français telles qu'elles s'expriment dans l'œuvre collective La civilisation surréaliste (Payot 1976), publiée sous la direction du surréaliste parisien Vincent Bounoure, ancien ami d'André Breton depuis le milieu des années 1950. La contribution à cet ouvrage de Vratislav Effenberger, animateur du groupe pragois après la mort de Karel Teige, a également été majeure. Face au silence général observé sur ce travail par les experts du surréalisme et de la sémiotique, nous nous pencherons sur le nouveau jeu collectif des récits parallèles, inventé dans les années 1970, et l'actualité des réflexions surréalistes sur le cours pris par l'usage du langage dans nos civilisations par le biais de la communication de masse, soumettant l'échange de signes à la logique de l'économie capitaliste et aux objectifs idéologiques du pouvoir politique. Par leur expérience ludique et poétique, les surréalistes ont compris le tournant négatif que prenait l'« universalité » du langage et de l'art. Ces idées sont confirmées et approfondies par quelques lettres et documents d'archives inédits, échangés entre Bounoure et Effenberger lors de la préparation du volume et jusqu'ici jamais pris en considération. Nous montrerons aussi l'existence de convergences implicites étonnantes entre cette sémiotique générale surréaliste et les théories des signes de Bakhtine et Rossi-Landi.

Mots clés : Surréalisme, récits parallèles, objets parallèles, homologie, sémiotique.

\section{Repères historiques et méthodologiques}

Dans le cadre de ce colloque de l'AILc consacré aux langues et aux langages qu'étudient et dont se nourrissent les études comparatistes, nous voudrions montrer l'intérêt de la réflexion sémiotique des poètes surréalistes français et tchèques au travers de l'ouvrage collectif La civilisation surréaliste, qu'ils ont réalisé en 1976. Il s'agit d'un sujet qui est au cœur des recherches que nous accomplissons depuis 15 ans désormais autour de la prétendue fin du « surréalisme historique » décré-

Ә Open Access. (c) 2021 Andrea D’Urso, published by De Gruyter. (c) BY-NC-ND This work is licensed under the Creative Commons Attribution-NonCommercial-NoDerivatives 4.0 International License. 
tée en 1969 par Jean Schuster et démentie par une série d'activités collectives et d'œuvres théoriques, poétiques et plastiques, que nous avons mises en lumière par nos travaux scientifiques, face à l'oubli plus ou moins volontaire où elles ont été reléguées par le paradigme dominant des spécialistes du surréalisme ${ }^{1}$.

On ne rencontrera pas de réserves quant au fait que Vratislav Effenberger est le véritable animateur du surréalisme tchèque après la mort de Karel Teige, dont il peut être considéré comme le continuateur. Au contraire, maintes objections s'élèveront si nous disons, comme nous osons le dire au vu de nos recherches, que c'est à Vincent Bounoure qu'on doit la continuation d'une activité surréaliste collective à Paris autour du Bulletin de liaison surréaliste (ou BLS, 1970-1976) après la mort de Breton, dont il a prolongé les théories et les enseignements. Vincent Bounoure fut également le maître d'œuvre de La civilisation surréaliste, réalisée en collaboration avec d'autres camarades français du BLS (Jean-Louis Bédouin, Bernard Caburet, Robert Guyon et Roger Renaud), avec certains membres du groupe surréaliste tchèque (Vratislav Effenberger, Martin Stejskal et Jan Švankmajer, eux-mêmes participant au BLS), mais aussi avec d'importantes personnalités ayant côtoyé le mouvement surréaliste du vivant d'André Breton (René Alleau, Robert Lebel et Jean Markale).

Malgré le silence des experts en surréalisme, sémiotique et études slaves, on ne saurait nier que les deux interlocuteurs principaux dans l'élaboration de cet ouvrage sont Bounoure, en tant que coordinateur général, et Effenberger, en tant qu'intermédiaire praguois s'exprimant en français grâce à l'aide de Ludvík Šváb et d'autres camarades tchèques, d'où le sens de synthèse collective de cet échange dont témoignent trois documents inédits sur la préparation de La civilisation surréaliste. Ils montrent plus que l'ouvrage lui-même la présence des deux tendances conflictuelles : celle du structuralisme du côté tchèque et celle du poststructuralisme du côté français. On en trouve de vagues traces dans certaines contributions de La civilisation surréaliste, par quelques références explicites à Baudrillard chez Caburet et à Jakobson chez Lebel, Caburet et Effenberger. Ces références offrent une première piste de comparaison entre deux approches distinctes que confirme et approfondit particulièrement le premier des quatre pôles du débat dans les documents préparatoires : le langage, l'échange, le pouvoir, la logique.

D'un côté, Effenberger tenait à reconnaître un rôle de grande importance à Jakobson - ce qui s'explique historiquement par l'étroit lien existant entre le surréalisme tchèque et le Cercle linguistique de Prague par le truchement de Karel

1 Il n'y a pas lieu ici de revenir sur l'occultation de cette histoire de 1969 à nos jours dont rendent compte en détail notre thèse de doctorat de 2009 et nos nombreux travaux scientifiques. Pour un début de discussion de la bibliographie disponible sur ce sujet, $c f$. D’Urso (2013). 
Teige. De l'autre, Bounoure tenait à reconsidérer ce rôle, en s'appuyant sur la critique que Baudrillard avait proposée - et que les Tchèques ne connaissaient pas - de la connivence de la théorie structuraliste de la communication avec la théorie de l'information et donc avec la pratique dominante du langage à code unique du monde contemporain. Ainsi, Effenberger ne semble pas saisir la perspective de Bounoure, que nous définirions comme étant de sémiotique générale ; ce dernier semble s'apercevoir lui-même de ce décalage, quand il croit reconnaître deux approches : l'une « sociologique » du groupe praguois, l'autre « subjective » du groupe français, qui devraient à son sens se rencontrer, si possible ${ }^{2}$.

Cette dialectique $d u$ " subjectif » et $d u$ " sociologique », du singulier et $d u$ collectif est à la base de La civilisation surréaliste. Témoignant donc de l'effervescence du surréalisme français et tchèque encore dans les années 1970, cet ouvrage montre que le langage y est présent à trois niveaux entrelacés : le poétique, le théorique et l'idéologique. Regardons-les de plus près.

\section{La poétique des « récits et objets parallèles » au vu de la sémiotique de Bakhtine}

Quand nous disons poétique, nous pourrions dire aussi ludique car nous nous référons au « langage surréaliste » tout court, cet ouvrage proposant une réflexion à partir de l'expérience d'automatisme collectif initiée en 1970 par l'un des nouveaux jeux inventés et pratiqués par les surréalistes du BLS : le jeu des « récits parallèles ", dont la place dans la relance des activités surréalistes est jusqu'à présent ignorée par la critique. Nous avons pu déjà le résumer ainsi :

Bref, il consiste à proférer à tour de rôle, et au bout d'un laps de temps préétabli, un mot que tous les participants sont tenus d'insérer dans leur propre acte d'écriture automatique en cours. Le résultat est justement constitué par autant de récits que de présents, et chacun contient tous les termes prononcés par chaque joueur, mais insérés dans des contextes différents suivant le devenir de l'écriture de chacun d'entre eux. (D’Urso 2011, 6)

2 Les trois textes inédits conservés dans les archives de la famille Bounoure sont : Bounoure (1973), Effenberger (1974) et Bounoure (1974). Ces documents sont l'un des exemples du débat qui a toujours existé au sein du surréalisme. Les dissemblances existant entre les deux groupes surréalistes dans l'approche théorique n'empêchent ni leur unité au niveau international ni leur accord sur les principes inspirateurs et les activités collectives du mouvement. C'est dire que la critique spécialisée, relevant des dissemblances souvent évoquées pour singulariser et séparer chaque groupe surréaliste dans le monde, sous-estime une synergie unitaire au vu d'anciens liens trop aisément négligés au détriment de la dialectique propre du mouvement. 
Des exemples des résultats de ce jeu se retrouvent déjà dans le $B L S$ et sont reproduits aussi dans La civilisation surréaliste, en signe d'une unité retrouvée dans la différence, mais aussi d'une alternative " polysémique » au code univoque et établi qui régit la communication dans la société contemporaine. Il y en a déjà assez pour que cet ouvrage mérite davantage d'attention que le silence avec lequel il a été accueilli. Mais il y a plus. S’en tenant à la tonalité affective dictée par l'automatisme individuel, les surréalistes ont vérifié par leurs jeux ce que Bakhtine $(1977,116)$ exprimait de la sorte : « c'est la pluralité d'accents du mot qui rend celui-ci vivant. Le problème de la pluri-accentuation doit être lié étroitement à celui de la polysémie ${ }^{3}$ ». Et d'ajouter : «La multiplicité des significations est l'indice qui fait d'un mot un mot » (Bakhtine 1977, 144).

Bakhtine (1977, 142-143, 147) est d'autant plus précis sur ce point qu'il considère tout signe comme le résultat d'une triade : le thème ("individuel et non réitérable. Il se présente comme l'expression d'une situation historique concrète ayant donné naissance à une énonciation », et il est « concret comme cet instant historique auquel l'énonciation appartient ») ; la signification au sens strict (« un appareil technique de réalisation du thème » dont les éléments sont "réitérables et identiques ») ; et l'accent de valeur ou appréciatif (sans lequel " il n'y a pas de mot »). Ce dernier recoupe ce qu'Effenberger $(1974,2,7)$ considère comme « l'orientation valorique, de nature déjà entièrement idéologique », précisant ainsi que « la contradiction dans les orientations valoriques nous semble représenter une source stimulante de la pensée humaine ».

Le jeu surréaliste des récits parallèles montre les usages et les contextes multiples dans lesquels pourrait se trouver le même mot, ses significations étant à chaque fois redéfinies par les thèmes (pour reprendre ainsi les termes de Bakhtine) de l'énonciation concrète (le récit) de chacun des joueurs suivant aussi l'accent de valeur qu'ils confèrent aux mots communs. Au vu de cette expérience ludique collective, les surréalistes en viennent à considérer l'échange communicatif comme la rencontre de différents monologues qui ont lieu dans d'incessants discours souterrains de chaque individu. À ce propos, c'est encore Bakhtine (1977, 138, 123-124) qui a affirmé : « L'énonciation actualisée est comme une île émer-

\footnotetext{
3 La traduction de cet ouvrage de Bakhtine-Volochinov est donc postérieure à La civilisation surréaliste. Nous sommes fondés à croire qu'il n'était même pas connu des surréalistes français dans son édition anglaise parue en 1973, également postérieure à l'invention du jeu des récits parallèles en 1970 à Paris. Du moins, nous n'en avons trouvé aucune référence dans les archives consultées. En raison des vicissitudes de l'ouvrage de Bakhtine-Volochinov, débattues aujourd'hui encore, et de la censure imposée au groupe tchécoslovaque, il est difficile d'affirmer qu’à Prague, on connaissait déjà l'original russe.
} 
geant d'un océan sans limites, le discours intérieur »; et encore : « Le mot est une sorte de pont jeté entre moi et les autres ${ }^{4}$ ».

Bien que Bakhtine écrive cela en parlant de « l'interaction verbale », rien n’empêche de chercher des convergences avec l'expérience poétique des surréalistes. Dans le jeu des récits parallèles, en fait, chaque joueur offre à tour de rôle un mot aux autres, qui le reçoivent, pour qu'ils l'emploient librement dans leur propre acte d'écriture automatique. Qui plus est, dans sa variante qui a donné lieu aux dialogues de 1973, les surréalistes se placent plus encore sur le terrain de « l'interaction verbale » dont parle Bakhtine, où le mot n'est pas seulement un « pont » comme l'affirme ce dernier, mais aussi un « tremplin », comme l'écrivait Breton en 1924 : « Les mots, les images ne s'offrent que comme tremplins à l'esprit de celui qui écoute », à ceci près que, dans les dialogues surréalistes de 1973, il ne s'agit plus de poursuivre " simplement son soliloque » (Breton 1988 [1924], 336). L'énonciation de chaque individu qui actualise à l'extérieur son propre monologue intérieur s'insère dans une dynamique collective, dans ce qui se bâtit comme une « interaction verbale » précisément, une conversation ou un dialogue automatiques, où les répliques des interlocuteurs ne sont pas dictées, suivant un enchaînement logique ordinaire, par la raison, mais reposent plutôt sur la première phrase venue à l'esprit.

Cette approche ludique et poétique des signes ne saurait se borner aux mots : c'est dire que ce que nous appelons la sémiotique générale qu'ébauchent les surréalistes ne se limite pas à considérer le langage verbal, sinon il serait impropre de la nommer ainsi, car elle ne serait qu'une linguistique voire une glottologie ; mais plutôt, elle s'applique aussi au langage non-verbal. De fait, c'est par la transposition du jeu des récits parallèles au domaine des choses que naît le jeu des " objets parallèles » montrant même sur le plan plastique l'existence de cette polysémie évoquée plus haut, comme l'explique J.-L. Bédouin (1976, 296-297) dans une de ses contributions à La civilisation surréaliste. Cette variante du jeu collectif a donné lieu à des assemblages multiples et différents à partir des mêmes objets d'usage quotidien offerts à l'imaginaire des joueurs. Et Bounoure et Effenberger (1976c, 312) d'ajouter : «l'imaginaire est ce qui établit un rapport réel avec l'objet ; et ce rapport est transformation du monde ».

4 Pour approfondir ces sujets, $c f$. D’Urso (2014). 


\section{Les affinités de La civilisation surréaliste avec la sémiotique de Rossi-Landi}

Nous accédons par là au niveau théorique ou métalinguistique que nous annoncions au début et où se trouve de nouveau transposé par La civilisation surréaliste le jeu des récits parallèles, qui est à son origine. En effet, dans cet ouvrage le discours sémiotique sur le langage se présente comme un recueil de « réflexions parallèles » des surréalistes sur le sort réservé par la société contemporaine à la communication humaine en particulier et à l'échange de signes en général, de plus en plus soumis, l'une et l'autre, à l'idéologie du pouvoir politique et à la logique de l'économie marchande. En d'autres termes :

Sur le plan de La civilisation surréaliste, le jeu devient une occasion pour réfléchir sur des sujets communs, mais chacun de sa propre façon, en développant des discours qui se recoupent dans certains points pour ensuite continuer sur des routes différentes menant à de multiples critiques, approfondissements et suggestions qui concernent la société contemporaine. Une certaine répétitivité que sans aucun doute on peut relever dans ce volume est justement due à son principe directeur : certains arguments se représentent dans plusieurs contributions, de même que dans le jeu des récits parallèles reviennent les mots prononcés à voix haute. (D’Urso 2011, 6)

Pour tenter de résumer les 346 pages de La civilisation surréaliste, nous dirions qu'Effenberger et Bounoure (1976a, 25) dénoncent une hypertrophie des systèmes sociaux et économiques dans « l'évolution générale des sociétés par formalisation aberrante », là où « le nom de toutes parts désormais excède la chose ; l'organe excède sa fonction réelle et cesse de la remplir ». "Cette dégénérescence de la fonction dans un symbole gigantisé recouvre approximativement le passage historique de la valeur d'usage à la valeur d'échange ", ce par quoi tout ressort psychologique sur lequel se fondent les activités humaines, la communication et l'expression est dérouté et exploité « pour les soumettre aux lois économiques de l'échange mercantile et en évacuer toute valeur d'usage » : c'est ce que démontrent bien les arts plastiques, dont la valeur de « communication affective » est détournée par un « code commercial » qui en règle la circulation et en fixe la valeur «par convention d'échange » (Bounoure et Effenberger 1976b, 67-68). Pour Effenberger (1976), sur le plan du langage en général, l'application du système dominant de valeurs du discours économique, qui se fonde sur des critères commerciaux, mène à la réduction des signes à une seule de leurs fonctions : l'échange utilitaire d'informations. Caburet (1976b, 164) rappelle que la pensée surréaliste recoupe ici les analyses du Baudrillard dans La Société de consommation (1970) et Pour une critique de l'économie politique du signe (1972) sur l'abstraction croissante des valeurs et la fétichisation des objets-signes. 
En s’appuyant sur l'ethnologie de Mauss et Malinowski, repris par Bataille et Baudrillard, Bounoure souligne que l'unanimité des économistes à l'égard du surplus comme origine de l'échange procède, en réalité, d'un ethnocentrisme négligeant les cas où les faits économiques révèlent leur aspect de communication et où la circulation de matières et artefacts a pour fonction de garantir de bons rapports intersubjectifs et l'ouverture du canal nécessaire à leur entretien, en dehors de tout équilibre entre les valeurs d'échange, bien au contraire. C'est ce que démontrent la Kula en Nouvelle-Guinée orientale et le potlatch en Colombie-Britannique. À cette valeur de discours de l'échange économique, l'utilitarisme de la civilisation de l'homo œconomicus a substitué les chiffres des Grands Livres : la perte des relations sociales qui va avec concourt à créer des « agrégats humains qu'il faudrait nommer des non-civilisations » (Bounoure 1976d, 154).

« Le traité d'économie qui pourrait s'esquisser dans les points de suspension de La Civilisation surréaliste ", comme l'écrit Bounoure, ne se limite donc pas à une dénonciation de l'asservissement de l'être aux choses, mais il propose « que les choses retrouvent, elles aussi, dans l'interrelation économique, leur sens, ce sens retrouvé sur le dernier versant $\mathrm{du} \mathrm{XIX}^{\mathrm{e}}$ siècle dans l'interrelation verbale plus communément désignée sous le nom de poésie moderne » (Bounoure 1976c, 143-144, c'est nous qui soulignons). Pour lui, la considération, marxiste même, d'usage et échange comme valeurs insère l'un et l'autre dans " une dialectique abstraite où disparaissent les orientations vivantes de leur conjugaison pratique », cela portant à la codification totalitaire de l'usage des objets selon une fonction univoque, car leur sens « est prédéterminé par le discours économique » de leur propre matrice de transformation industrielle, qui leur retire toute polysémie foncière (144-146). Donc, la relation de l'homme aux choses en sort altérée : « l'intervention humaine n'est qu'un travail sur les choses : la servitude de la matière, à grand-peine obtenue, a pour corollaire la servitude humaine » (146, c'est nous qui soulignons). Si par le jeu des récits parallèles le surréalisme a expérimenté que la fécondité du discours « est directement liée à la transformation sémantique opérée par l'échange et aux interrelations qu'elle autorise entre chaînes matricielles distinctes, l'interrelation économique et la liberté dans l'ordre pratique apparaissent sujettes aux mêmes lois » (146, c'est nous qui soulignons). C'est pourquoi les mots et les choses réduits à un véhicule du code dominant doivent être libérés, car de leur liberté dépend celle de l'homme (147). Le mot suivant de Bounoure résume au mieux la perspective surréaliste jusqu’ici énoncée :

Dans la pratique, ce qu'on appelle les sociétés humaines consacrant une assez large part de leur activité à des relations d'échange ou à des relations de pouvoir, ces faits forment un chapitre considérable de leur langage : langage certes « infrastructurel », mais qui échappe par là aux accusations de futilité dont restent marquées les "superstructures ». Cette activité doit être rendue maintenant à son sens véritable, qui est la libre expression des 
structures individuelles dans la transformation de matériaux d'usage public : les mots, les marchandises, les statues. [...] De la possibilité d'une autre infrastructure, où le travail est libre parole, au lieu de signifier la servitude, dépend réellement le « désencroûtement des mœurs » que réclamait Breton, dépend la mise à mort d'une mythologie mortelle, dépend la subversion entière du rapport de l'homme avec le monde et des rapports entre hommes. (Bounoure 1976b, 111 ; c'est nous qui soulignons)

Ce propos recoupe d'une manière étonnante la sémiotique marxiste que le philosophe italien du langage Ferruccio Rossi-Landi développait entre les années 1960 et 1970. Par son homologie de la production linguistique et de la production matérielle, il proposait de considérer le langage en général comme travail et toute langue historico-sociale comme à la fois le produit, le matériau et le moyen de ce travail. La langue constituant ainsi ce qu'on peut appeler le capital (linguistique) fixe, les gens qui parlent telle ou telle langue sont alors le capital (linguistique) variable, les travailleurs linguistiques ou la « marchandise parlante». Rossi-Landi pousse son homologie au niveau du marché, c'est-à-dire de la communauté linguistique, où les messages circulent comme des marchandises et les marchandises comme messages ${ }^{5}$. Si cela fait déjà voir toute la proximité qui existe entre l'intuition sémiotique des surréalistes et la perspective rossi-landienne, cette autre remarque de Bounoure la confirme davantage : « Désormais il est possible d'envisager la production des paroles, leur stockage, leur consommation, d'évaluer leur valeur d'usage et leur valeur d'échange suivant les lois générales qui règlent dans le métabolisme social la transformation des biens matériels » (Bounoure 1976e, 168, c'est nous qui soulignons).

C'est à plus forte raison dans les documents inédits sur la préparation de $L a$ civilisation surréaliste qu'on trouve l'étonnante affinité avec la terminologie rossi-landienne, lorsque Bounoure écrit à Effenberger :

Or il nous semble qu'il faut désormais restituer à toute circulation de marchandise son sens originel et sa valeur de discours qui s'échange, discours obéissant aux lois de tout discours, c'est-à-dire pourvu d'une structure propre, dérivant d'un procès de production particulier, usant de matières communes comme sont communs à tous les mots dans les Récits parallèles, mais les inclinant à des fins particulières. (Bounoure 1973, 3 ; c'est nous qui soulignons)

De même, par l'idée que « l'univocité de la relation signe-sens est un leurre [...] qui ruine la communication interhumaine ", et qu' " on commence à se comprendre quand on sait que le sens du mot (comme la valeur de la marchandise) vient du

5 Pour un aperçu en français, $c f$. Rossi-Landi (1973 ; 1969 [1967] ; 1974 [1973]). Rien ne prouve que les surréalistes français connaissaient ces textes à l'époque. 
“travail général abstrait” que lui a incorporé la matrice où il a été mis en œuvre ", (Bounoure 1974, 4, c'est nous qui soulignons) confirme toute la latitude que peut prendre la convergence de la vision surréaliste avec la sémiotique de Rossi-Landi.

\section{Le langage poétique comme préparation « idéologique » de la révolution}

Pour ce qui est du niveau idéologique de La civilisation surréaliste évoqué d'entrée de jeu, c'est encore au sens rossi-landien de « l'idéologie comme vision du monde et projet de société » qu'il faut l'entendre (Rossi-Landi 2005 [1978]). Aucun sémiologue n’a considéré ces liens possibles, que renforcent les remarques de Bédouin (1976a), sur la société programmée et de Caburet (1976a) sur le rôle paradoxal du langage d'enchaîner et de libérer l'homme à la fois, en le rendant soit un « usager » esclave de la publicité, du marketing et du mythe de la consommation des industries que sont devenues désormais la communication et l'information, soit un insoumis qui se révolte contre cette logique utilitaire par son propre langage poétique. Bounoure $(1973,5)$ en vient aussi à reconsidérer « la notion de pouvoir comme obligation faite au public d'user d'un code privé ».

Ce sont exactement les problèmes de « l'exécution des programmes sociaux », de "l'aliénation linguistique », de la non-liberté de l'usage de la langue et de la " propriété privée de la langue (qui est publique) » qu'a envisagés Rossi-Landi (1972 [1967], 241 ; 1972 [1968], 219), redéfinissant ainsi la classe dominante comme la classe qui possède les moyens de communication (codage, circulation et décodage des messages, redondance, bruit et brouillage de l'information), et les classes subalternes comme celles qui « en subissant la classe dominante se limitent à en employer les codes ou sinon se taisent » (Rossi-Landi 1972 [1968], 220 ; 1975, 183-194). D’où le propos qu'« opérer sur les systèmes fondamentaux de signes qui régissent une société signifie préparer idéologiquement la révolution » (Rossi-Landi 1972 [1969], 339), et que « si on veut changer d'idéologie, il faut changer de pratique sociale » (Rossi-Landi 2005 [1978], 97).

Ces devises rossi-landiennes font pendant aux idées de Bounoure $(1973,4,5)$ affirmant « que "l'idéologie dominante" exerce, à l'instar d'une véritable mythologie, un pouvoir plus redoutable que celui-même de la police », et qu' « il n'est pas de fait où se manifeste le pouvoir qui ne procède d'une mythologie ou d'un discours antérieur ». Ou encore, comme l'écrit Effenberger $(1974,5)$ : « La parole elle-même ne devient un instrument du pouvoir que si elle correspond à une faim mythologique ou idéologique et qu'elle soit capable de l'assouvir de manière illusoire ». Par son dessein d'appliquer la nouvelle théorie surréaliste du langage, 
découverte par le jeu des récits parallèles, à l'intégralité des faits sociaux, y compris les faits économiques, vu l'importance qu'ils revêtent dans la société d'aujourd'hui, Bounoure (1973, 3), tout en s'approchant de Rossi-Landi sans le savoir, en renverse l'issue, contre tout économisme, et il marque également une distance à l'égard de Baudrillard. En fait, la considération surréaliste de l'économie comme un langage n'implique ni un remplacement ni un refus de l'application au langage de l'analyse marxiste de l'économie que propose Rossi-Landi ; bien au contraire, elle la complète dialectiquement, en ne se limitant pas à la « critique de l'économie politique du signe », comme chez Baudrillard. La conclusion de Bounoure est effectivement que « l'économie politique est à réécrire » (Bounoure 1973, 4, c'est nous qui soulignons).

Cela ne dément pas le propos de Rossi-Landi quant à la nécessité de travailler à l'aide d'une praxis révolutionnaire et désaliénante, afin d'atteindre une société fondée sur le jeu, à ceci près que, pour les surréalistes, les pratiques ludiques et poétiques (dont Rossi-Landi 1975, 182-183, n'ignore pas le rôle de libération) sont partie prenante de ce projet social de désaliénation, qui est pour eux précisément aussi un projet pour une civilisation autre. En fait, en 1978, Bounoure fut interpellé dans un débat de Critique communiste suscité par Michel Lequenne et Michael Löwy, qui s’alarmaient du remplacement de la formule de « révolution surréaliste » de l'époque de Breton par celle de « civilisation surréaliste » que proposait cet ouvrage collectif. Bounoure répondit que «S’il s’agit de faire la révolution, c'est bien pour arriver à une civilisation » (Bounoure et al. 1978, 101).

Encore à propos de mystification idéologique, nous voudrions consacrer notre dernier mot à l'absurde a priori universaliste attribué à la poésie ou aux « arts comme code universel ». La critique faite par La civilisation surréaliste nous apprend que l'acception de « code universel » n'est pas toujours positive : elle recèle des partis pris, des préjugés, des pensées dirigées ayant comme contrepartie une idéologie, c'est-à-dire des pratiques sociales et un projet de société à même d'entacher d'obscurité et d'inintelligibilité tout ce qui demande un effort supérieur de découverte et de compréhension. À ce propos, en polémiquant avec un texte de Marcuse sollicité par les surréalistes américains en 1973, Bounoure (1999 [1973], 128) répliqua que « La parole poétique, quand elle surgit, n’a pas d'auditoire prédestiné. Elle n'est qu'un cri. Elle ne cherche pas à convaincre. Elle s'élève dans la certitude d'être sans partage. [...] L'universalité est un accident historico-sociologique de la lecture du poème : elle est étrangère à sa création ».

Songeons à la poétique de La civilisation surréaliste elle-même : elle nie l'universalisme impérialiste du code unique et établi ; elle rétablit l'échange et les rapports sociaux qui en découlent sur la rencontre de discours parallèles indépendants, en restituant ainsi au langage sa valeur expressive et à la parole échangée sa fonction de poétiser la vie collective. Quoi d'incompréhensible, d'obscur, 
voire d'inexact dans ces propos ? Leur actualité brûlante ne les rend-elle pas plus encore " universels » ? Et pourtant cet ouvrage n'a pas parlé à d'autres comme il nous parle à nous, ce qui s'explique aisément historiquement et sociologiquement. Il n'en reste pas moins que La civilisation surréaliste est la pierre de touche permettant de comprendre le surréalisme français et tchèque après la mort d'André Breton et de Karel Teige, par la voix de leurs plus grands continuateurs : Vincent Bounoure et Vratislav Effenberger, qui parlent aujourd'hui encore à celles et ceux qui veulent les entendre.

\section{Références bibliographiques}

Bakhtine, Mikhaïl (V. N. Volochinov). Le Marxisme et la philosophie du langage. Essai d'application de la méthode sociologique en linguistique [1929]. Trad. de Marina Yaguello, introd. de Roman Jakobson. Paris : Éditions de Minuit, 1977.

Bédouin, Jean-Louis. «Information, communication, quadrillage et ratières ». La civilisation surréaliste. 1976a. 82-95.

Bédouin, Jean-Louis. «Cycle de l'objet ». La civilisation surréaliste. 1976b. 295-302.

Bounoure, Vincent. "Sur les tâches immédiates du surréalisme ». Inédit : archives Bounoure, s. d. (fin 1973), 6 pages dactylographiées.

Bounoure, Vincent. "D’après remarques ». Inédit : archives Bounoure, s. d. (février-mars 1974), 9 pages dactylographiées.

Bounoure, Vincent. «Libre échange avec Herbert Marcuse ». Bulletin de liaison surréaliste 7 (1973) : 11-18 ; reproduit dans Moments du surréalisme. Paris, L’Harmattan, 1999. 125-134.

Bounoure, Vincent (éd.). La civilisation surréaliste. Paris : Payot, 1976a.

Bounoure, Vincent. « Panorama ». La civilisation surréaliste. 1976b. 110-112.

Bounoure, Vincent. «Solve et coagula ». La civilisation surréaliste. 1976c. 143-147.

Bounoure, Vincent. «Généalogie de l'échange ». La civilisation surréaliste. 1976d. 148-156.

Bounoure, Vincent. «Échange et communication ». La civilisation surréaliste. 1976e. 168-173.

Bounoure, Vincent, et Vratislav Effenberger, « De 24 à 75 ». La civilisation surréaliste. 1976a. 24-27.

Bounoure, Vincent, et Vratislav Effenberger. «Le cri, le chant, le renseignement ». La civilisation surréaliste. 1976b. 67-72.

Bounoure, Vincent, et Vratislav Effenberger. « Art magique et révolution ». La civilisation surréaliste. 1976c. 310-313.

Bounoure, Vincent, Lequenne, Michel et Carlos Rossi. «À propos de la Civilisation surréaliste ». Critique communiste 24 (1978) : 100-126 ; reproduit dans Bounoure, Vincent. L'événement surréaliste. Paris : L'Harmattan, 2004. 134-164.

Breton, André. « Manifeste du surréalisme » [1924]. Euvres complètes, t. I. Paris : Gallimard, 1988. 309-346.

Caburet, Bernard. «Chaînes parlées et prisons linguistiques ». La civilisation surréaliste. 1976a. 96-103.

Caburet, Bernard. «Injonction du désir ». La civilisation surréaliste. 1976b. 160-167. 
D’Urso, Andrea. «Poésie, peinture, sémiotique et anthropologie chez Vincent Bounoure (ou De quelques limites de la critique littéraire) ». Between 1.1 (2011). http://ojs.unica.it/index. php/between/article/view/195/231 (27 avril 2018).

D’Urso, Andrea. «Un surréaliste méconnu : Vincent Bounoure et la critique française et italienne ».Publif@rum 19 (2013). https://www.publifarum.farum.it/ezine_pdf.php?art_ id=269 (27 avril 2018).

D’Urso, Andrea. « Bakhtine, Morris, Rossi-Landi et le surréalisme : pour une sémiotique matérialiste-dialectique des valeurs du signe ». Semat 2.1 (2014) : 195-207. http:// journals.uob.edu.bh/SEMAT/contents/volume-13/articles/article-127 (27 avril 2018).

Effenberger, Vratislav. "Quelques remarques sur les tâches immédiates du surréalisme ». Inédit : archives Bounoure, 15 janvier 1974, 7 pages dactylographiées.

Effenberger, Vratislav. « L'économie est-elle un discours ? ». La civilisation surréaliste. 1976. 137-138.

Rossi-Landi, Ferruccio. «Extension de l'homologie entre énoncés et outils » [1967]. Actes $d u x^{\mathrm{e}}$ Congrès international des linguistes, t. i. Bucarest : Éditions de l'Académie de la République Socialiste de Roumanie, 1969. 503-508.

Rossi-Landi, Ferruccio. "Sui programmi della comunicazione non-verbale » [1967]. Semiotica e ideologia. Milan : Bompiani, 1972. 237-251.

Rossi-Landi, Ferruccio. «Capitale e proprietà privata nel linguaggio » [1968]. Semiotica e ideologia. Milan : Bompiani, 1972. 201-227.

Rossi-Landi, Ferruccio. " Dialettica e alienazione nel linguaggio. Colloquio con Enzo Golino » [1969]. Semiotica e ideologia. Milan : Bompiani, 1972. 253-339.

Rossi-Landi, Ferruccio. "Le langage comme travail et comme marché ». L'homme et la société 28 (1973) : 71-92.

Rossi-Landi, Ferruccio. «Sur l'argent linguistique» [1973]. Psychanalyse et politique. Armando Verdiglione (éd.). Paris : Le Seuil, 1974. 103-127.

Rossi-Landi, Ferruccio. Linguistics and Economics. La Haye et Paris : Mouton, 1975.

Rossi-Landi, Ferruccio. Ideologia [1978, 1982]. Augusto Ponzio (éd.). Milan : Meltemi, 2005.

Andrea D’Urso received his MPhil (2008) and PhD (2009) in Comparative Literature and Literary Text Translation from the University of Siena with the "Doctor Europaeus" label. His $\mathrm{PhD}$ thesis, considered a pioneer work, was submitted under the title Vincent Bounoure and the developments of Surrealism after André Breton's death. He is now a lecturer in French Language and Literature at Salento University (Italy), and in Italian Language and Literature at SHS University in Lille (France). In addition to his numerous articles (including those on Walter Benjamin, Nicolas Calas and Pierre Mabille), he is the author of Théorie et écritures surréalistes (with A. Calì, Pensa 2012). He is also known as one of the few specialists and successors of Ferruccio Rossi-Landi's semiotics. He is currently writing a research thesis at SHS University in Lille on the promoter of Italian free verse, Gian Pietro Lucini. 